\title{
The influence of material density on bipolar charge transport in polymer-based electronic applications
}

\author{
Marta M. D. Ramos ${ }^{1, a}$, Helena M. G. Correia ${ }^{1, b}$ and Hugo Carmo ${ }^{1, c}$ \\ ${ }^{1}$ Departamento de Física, Universidade do Minho, Campus de Gualtar, \\ 4710-057 Braga, Portugal \\ amarta@fisica.uminho.pt, ${ }^{b}$ hcorreia@fisica.uminho.pt, ${ }^{\circ}$ hugo carmo@yahoo.com
}

Keywords: mesoscopic modelling, density effect, charge transport, semiconducting polymers.

\begin{abstract}
Using a mesoscopic modelling approach, the authors performed computer experiments to study the influence of polymer density on bipolar charge evolution through thin layers of polydiacetylene (PDA) exhibiting specific microstructures. We found that the competition between charge transport, trapping and recombination within the polymer layer leads to several general trends, some of them being non-intuitive, as one varies polymer density. Our results show that polymer density mainly affects current and recombination efficiencies in the absence of defects or impurity states. The overall trends depend both on chain orientation relative to the electrodes and on the strength of the external applied electric field. These results suggest that adequate modelling of charge transport in electronic and optoelectronic devices based on conducting and semiconducting polymers, such as PDA, must include their structure and related key factors at mesoscopic scale. Such models provide the necessary knowledge-base to optimize the polymer film structure for electronic applications.
\end{abstract}

\section{Introduction}

Electronically-active polymers have found potential applications in fabrication of Schottky diodes, light-emitting diodes, solar cells and field-effect transistors [1-4]. The limitations of these polymer based devices are posed by the performance of the active polymer layer.

The polymer layer can be considered a network of highly conjugated polymer molecules with variable chain length and different orientations relative to the electrodes. There are clear indications that processing conditions affect the packing structure in the polymer layer, which is reflected in its density. The overall microstructure profoundly influences the bipolar charge transport in the polymer layer - which are intra-chain motion and inter-chain hopping between discrete localized levels of individual polymer molecules - and recombination throughout its entire volume. Clearly many factors are at play and the various effects have yet to be deconvoluted.

Computational techniques, in particular mesoscopic modelling and simulation, can help us to understand how each factor influences bipolar charge transport, trapping and recombination within the polymer film. The mesoscopic modelling technique developed by us [5], which includes both intramolecular and intermolecular charge transport through polymer networks as well as intramolecular recombination it is a suitable tool for this type of studies. The geometrical simplicity of polydiacetylene (PDA) makes it an ideal system to address these issues that should also apply to other semi-conducting polymers. We shall concentrate here on the effect of polymer density for three different polymer chain orientations relative to the electrodes and several applied bias voltage. 


\section{Mesoscopic model and simulation method}

Before any injection of charge into the polymer layer is considered, we built several realizations of a PDA network placing individual straight strands, with mean length of 5 monomer units and a Gaussian distribution of lengths with $1 /\left(2 \sigma^{2}\right) \sim 0.1$, in the gap between two planar electrodes with their long molecular axis parallel, perpendicular or randomly oriented relative to the electrode surface.

The number of straight strands is chosen to give a certain polymer density between two electrodes with an area of $400 \mathrm{~nm}^{2}$ localized at a distance of $100 \mathrm{~nm}$. Polymer densities ranging from 0.10 to $0.15 \mathrm{~g} / \mathrm{cm}^{3}$ were used in order to prevent non-physical overlaps between the straight strands.

When a bias voltage is applied, one electron and one hole are injected in the PDA network from opposite electrodes at polymer strands bound to the electrodes chosen randomly. After these charges hop to neighbour strands, another pair of charges of opposite sign is injected in the network. This sequence of events is repeated and a steady state is reached.

Before a charge can hop to another strand, it moves to the strand end favoured by the local electric field (which is the sum of the external applied electric field, the field created by the other charges within the polymer network and the field due to electrode polarisation) if its values is greater than the field needed to move charges along the PDA chains $\left(2.35 \times 10^{8} \mathrm{~V} / \mathrm{m}\right.$ for holes and $1.60 \times 10^{8} \mathrm{~V} / \mathrm{m}$ for electrons [6]) otherwise it moves towards the centre of the strand.

A charge is not allowed to hop to a neighbour strand if the local electric field is zero or the hopping rate is lower than $10^{-5}$. In the calculation of the hopping rate we consider the product of several factors [7]. One factor decreases exponentially with jumping distance, an other factor favours hopping in the direction of the local electric field and a third factor decreases exponentially with energy barrier between the strands involved in the hopping process (which depends on the difference between electron affinity for holes and ionization potential for electrons, as well as molecular charge and potential difference). The data for molecular properties used in these calculations were obtained from reference [6], and the dielectric constant of the polydiacetylene (2.3) used in the calculations of the local electric field was obtained from reference [8].

When a charge can hop to more than one neighbour strand, we assume that it hops to one of the strands for which the hopping probability is greater than $90 \%$, chosen randomly. Recombination is assumed to occur only when positive and negative charges meet on the same PDA strand.

\section{Results and discussion}

In the present work we consider the effect of polymer density on current, trapping and recombination efficiencies for three different molecular orientations relative to the electrodes: parallel, perpendicular and randomly oriented.

Fig. 1 shows the recombination predicted within the PDA film, for ten different network realisations and a balanced bipolar injection. Our results suggest that recombination decreases as density increases for polymer networks with no preferential orientation of their strands relative to the electrode surface regardless the strength of the external applied electric field.

Polymer networks with their strands parallel to the electrode surface show similar behaviour to the perpendicular for an external applied electric field greater than the threshold for intramolecular mobility of both electrons and holes. The overall behaviour for the three different microstructures (parallel, perpendicular and randomly orientations) is affected by the polymer density as well as by the strength of the external applied electric field. 


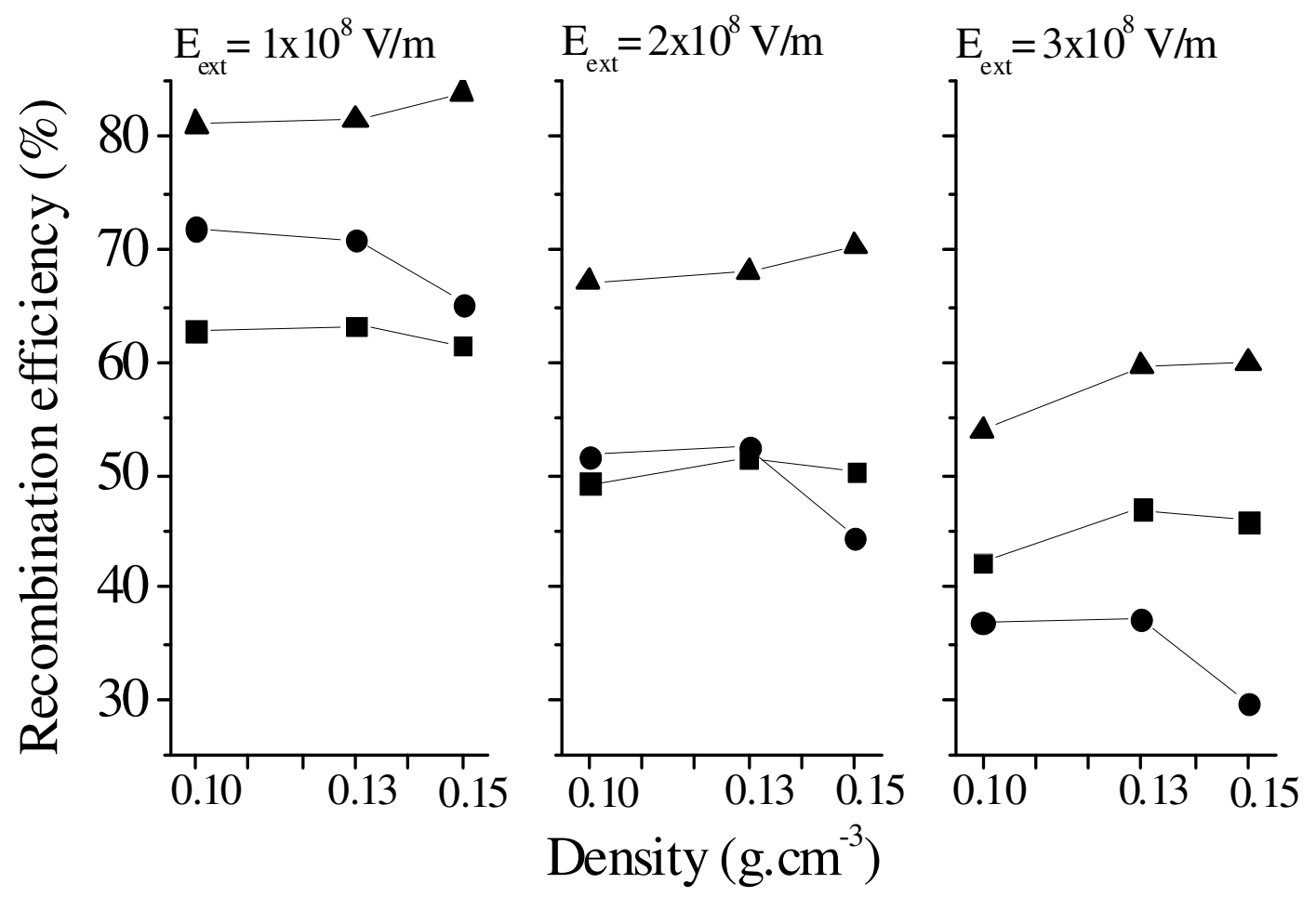

Fig. 1: The proportion of injected charge which recombine within the PDA network as a function of polymer density for three different external applied electric field, $\mathrm{E}_{\mathrm{ext}}$, and the following molecular orientation relative to the electrode surface: parallel (square), perpendicular (triangle) and random orientation (circle).

Fig. 2 illustrates the dependence of current efficiency (for electrons and holes) upon the polymer density for the same polymer structures mentioned above and the external applied electric field of $3 \times 10^{8} \mathrm{~V} / \mathrm{m}$. Since for the continuous injection of electron-hole pairs there is negligible charge trapping within the polymer layer (see Fig. 3), the current efficiency (proportions of injected charge which cross the polymer film to carry a current) shows an opposite behaviour to recombination.

Change in polymer density and applied bias voltage do not seem to produce any significant effect on charge trapping within the polymer layer for all polymer structures considered here. The greatest charge trapping predicted was for polymer structures with all strands normal to the electrodes. In this case, the electrons stored within the polymer layer increases slightly as polymer density increases whereas for holes it shows an opposite behaviour. 


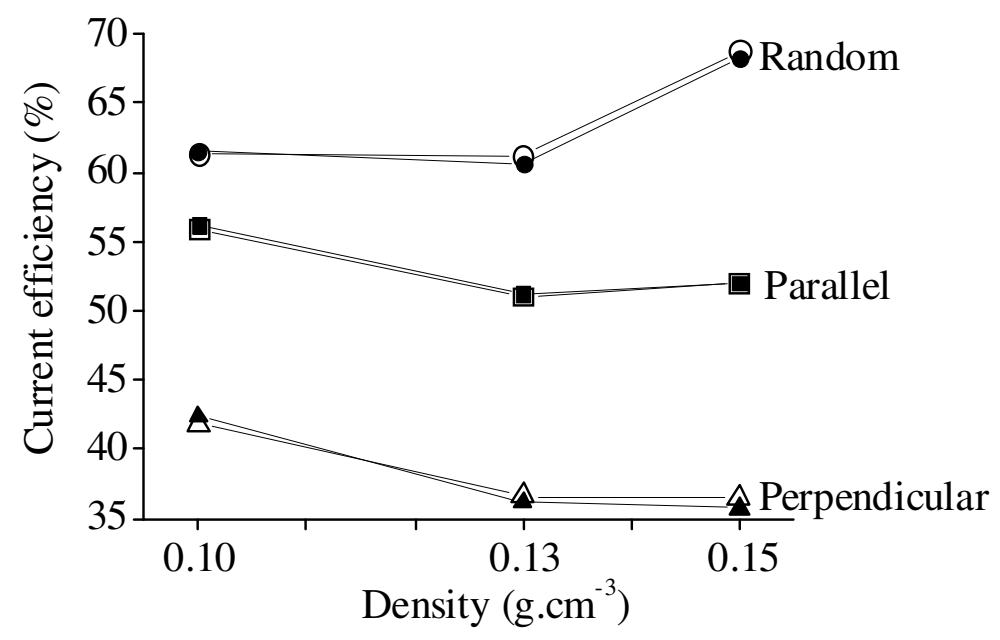

Fig.2: Current efficiency through the PDA network as a function of polymer density for: electrons (closed marks) and holes (open marks). These results were obtained using an external applied electric field of $3 \times 10^{8} \mathrm{~V} / \mathrm{m}$ and the polymer microstructures describe in the caption of fig. 1 .

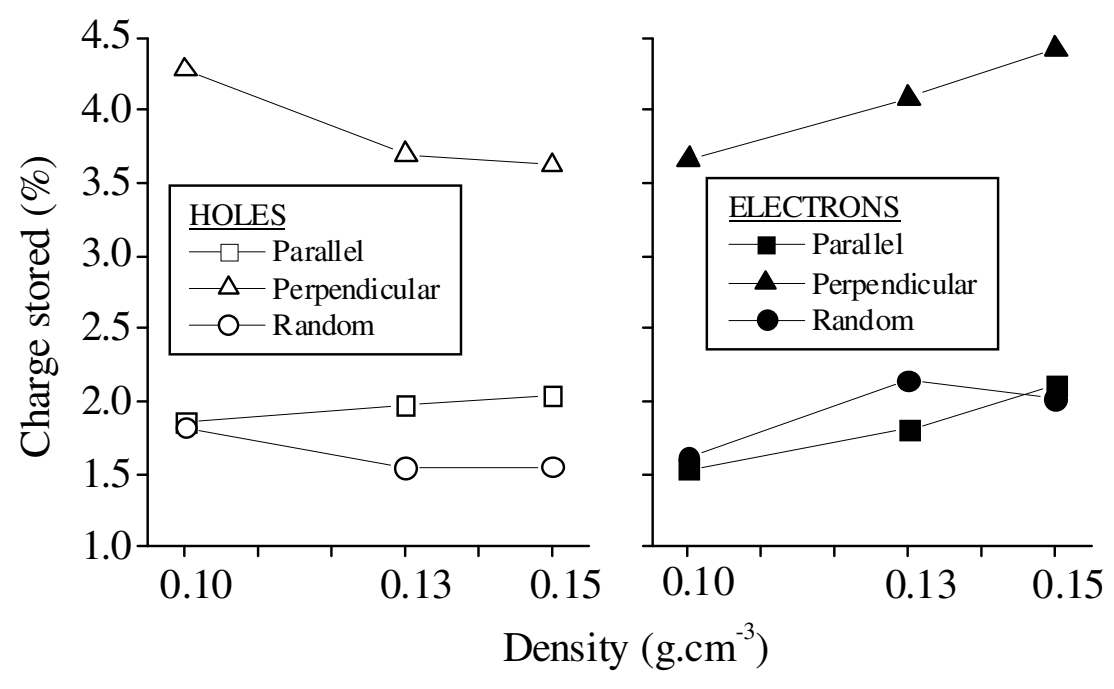

Fig. 3: The dependence of holes (left-hand side) and electrons (right-hand side) trapped at steady state within the PDA network on polymer density for the following molecular orientations relative to the electrode surface: parallel (square), perpendicular (triangle) and random orientation (circle). These results were obtained using an external applied electric field of $3 \times 10^{8} \mathrm{~V} / \mathrm{m}$.

\section{Conclusions}


The mesoscopic modelling of bipolar charge transport in polydiacetylene layer has proven to be a very successful method for obtaining insights into the effects of polymer density on the electronic behaviour of different polymer microstructures, which cannot be easily obtained experimentally. From our computer simulations for continuous injection of electrons and holes, we have found that polymer density mainly affects current and recombination efficiencies and has almost no effect on charge trapping in the absence of defects or impurity states. Moreover, the fact that there is

competition between trapping, transport and recombination within the polymer film leads to different trends, some being non-intuitive, for different polymer microstructures as one varies polymer density and the applied bias voltage.

\section{Acknowledgement}

This work is part of the research project POCTI/CTM/41574/2001, approved by the Portuguese Foundation for Science and Technology (FCT) and POCTI and supported by the European Community Fund FEDER. One of us (H.M.G.C.) is also indebted to FCT for financial support under PhD grant No. SFRH/BD/11231/2002.

\section{References}

[1] N. Sünel, A. G. Sedef, M. Parlak, and L.Toppare: Materials Chemistry and Physics Vol. 91 (2005), p. 227.

[2] J. H. Burroughes, D. D. C. Bradley, A. R. Brown, R. N. Marks, K. Mackay, R. H. Friend, P. L. Burns and A. B. Holmes: Nature Vol. 347 (1990), p. 539.

[3] G. Yu, J. Gao, J. C. Hummelen, F. Wudl and A. J. Heeger: Science Vol. 270 (1995) p. 1789.

[4] F. Garnier, R. Hajlaoui, A. Yassar and P. Srivastava: Science, Vol. 265 (1994), p. 1684.

[5] M. M. D. Ramos, H. M. G. Correia, R. M. Ribeiro and A. M. Stoneham: Synthetic Metals Vol. 147 (2004), p. 281.

[6] A. M. Stoneham, M. M. D. Ramos, A. M. Almeida, H. G. Correia, R. M. Ribeiro, H. Ness and A. J. Fisher: J. Phys. Condens. Matter Vol. 14 (2002), p. 9877.

[7] M. M. D. Ramos and A. M. Stoneham: Comput. Mater. Sci. Vol. 17 (2000), p. 260.

[8] T. Hasegawa, T. Okamoto; M. Haraguchi, M. Fukui, T. Koda and K. Takeda: Jpn. J. Appl. Phys. Vol. 37 (1998), p. 5793. 